\title{
Time course of the sensitivity and specificity of serum anti-SARS-CoV-2 IgM and IgG antibodies for the diagnosis of symptomatic COVID-19 in Japan
}

\section{Yuki Nakano}

Department of Clinical Laboratory, the University of Tokyo Hospital, Tokyo, Japan

\section{Makoto Kurano ( $\nabla$ kurano-tky@umin.ac.jp )}

Department of Clinical Laboratory, and , Graduate School of Medicine. the University of Tokyo Hospital, Tokyo, Japan

\section{Yoshifumi Morita}

Department of Clinical Laboratory, the University of Tokyo Hospital, Tokyo, Japan

\section{Takuya Shimura}

Department of Clinical Laboratory, the University of Tokyo Hospital, Tokyo, Japan

\section{Rin Yokoyama}

Department of Clinical Laboratory, the University of Tokyo Hospital, Tokyo, Japan

\section{Chungen Qian}

The Key Laboratory for Biomedical Photonics of MOE at Wuhan National Laboratory for Optoelectronics - Hubei

Bioinformatics \& Molecular Imaging Key Laboratory, Systems Biology Theme, Department of Biomedical Engineering,

College of Life Science and Technology, Huazhong University of Science and Technology, Hubei, P.R. China

\section{Fuzhen Xia}

Reagent R\&D Center, Shenzhen YHLO Biotech Co., Ltd, Guangdong, P.R. China

\section{Fan He}

Reagent R\&D Center, Shenzhen YHLO Biotech Co., Ltd, Guangdong, P.R. China

\section{Yoshiro Kishi}

Business Planning Department, Sales \& Marketing Division, Medical \& Biological Laboratories Co., Ltd, Tokyo, Japan

\section{Jun Okada}

Business Planning Department, Sales \& Marketing Division, Medical \& Biological Laboratories Co., Ltd, Tokyo, Japan

\section{Naoyuki Yoshikawa}

Department of Clinical Laboratory, the University of Tokyo Hospital, Tokyo, Japan

\section{Yutaka Nagura}

Department of Blood Transfusion, the University of Tokyo Hospital, Tokyo, Japan

\section{Hitoshi Okazaki}

Department of Blood Transfusion, the University of Tokyo Hospital, Tokyo, Japan

\section{Kyoji Moriya}

Department of Infection Control and Prevention, The University of Tokyo, Tokyo, Japan

\section{Yasuyuki Seto}

Department of Gastrointestinal Surgery, The University of Tokyo, Japan

\section{Tatsuhiko Kodama}

Laboratory for Systems Biology and Medicine, The University of Tokyo, Tokyo, Japan

\section{Yutaka Yatomi}

Department of Clinical Laboratory and Graduate School of Medicine, the University of Tokyo Hospital, Tokyo, Japan 


\section{Research Article}

Keywords: COVID-19, SARS-CoV-2, IgM, IgG

Posted Date: July 20th, 2020

DOI: https://doi.org/10.21203/rs.3.rs-44976/v1

License: (c) (i) This work is licensed under a Creative Commons Attribution 4.0 International License. Read Full License 


\section{Abstract}

The accurate and prompt diagnosis of SARS-CoV-2 infection is required for the control and treatment of the coronavirus infection disease 2019 (COVID-19). In this study, we aimed to investigate the time courses of the antisevere acute corona respiratory syndrome coronavirus 2 (SARS-CoV-2) IgM and IgG titers and to evaluate the sensitivity and specificity of such tests according to the specific day after the onset of COVID-19 among a patient population in Japan. We measured the titers of SARS-CoV-2 IgM and IgG in sera from 100 subjects, including 26 symptomatic COVID-19 patients, using chemiluminescent immunoassay (CLIA) methods utilizing magnetic beads coated with SARS-CoV-2 nucleocapsid protein and spike protein. The results of a ROC analysis suggested the possibility that the cutoff values in Japan might be lower than the manufacturer's reported cutoff (10 AU/mL): 1 $\mathrm{AU} / \mathrm{mL}$ for $\operatorname{lgM}$ and $5 \mathrm{AU} / \mathrm{mL}$ for IgG. The sensitivity of the test before Day 8 after symptom onset was less than $50 \%$; at Days $9-10$, however, we obtained a much higher sensitivity of $81.8 \%$ for both IgM and IgG. At 15 days or later after symptom onset, the SARS-CoV-2 IgG test had a sensitivity of $100 \%$. These results suggest that if the number of days since disease onset is taken into consideration, these antibody tests could be very useful for the diagnosis of COVID-19 and similar diseases.

\section{Introduction}

Since December 2019, the severe acute respiratory syndrome coronavirus 2 (SARS-CoV-2) has spread throughout the world. The accurate and prompt diagnosis of SARS-CoV-2 infection is required for the control of the COVID-19 pandemic as well as the initiation of adequate individual treatment. In clinical practice, the detection of SARS-CoV-2 RNA using a reverse transcriptase-polymerase chain reaction (RT-PCR) and nasopharyngeal swabs, saliva, or other upper respiratory tract specimens is considered to be the standard method for the diagnosis of COVID-19 ${ }^{1}$. Although the specificity of RT-PCR is relatively high, issues persist surrounding insufficient sensitivity (mainly because of the sampling technique), the timing of sample collection, and the types of specimens. Wang et al. reported that sensitivities of RT-PCR for bronchoalveolar lavage specimens, sputum, nasal swabs, fibrobronchoscope brush biopsy, pharyngeal swabs, feces, blood, and urine were $93 \%, 72 \%, 63 \%, 46 \%, 32 \%, 29 \%, 1 \%$, and $0 \%$ in a study of 205 COVID-19 patients ${ }^{2}$, and Pasomsub et al. reported that the sensitivity of RT-PCR using saliva samples was $84.2 \%$, compared with RT-PCR using nasopharyngeal and throat swabs ${ }^{3}$. The insufficient sensitivity of the RT-PCR method especially for nasopharyngeal swabs and saliva, which are commonly used specimens, sometimes produces false-negative results. Moreover, the sampling of respiratory and nasopharyngeal specimens can cause secondary infection.

Another method for detecting COVID-19 infection is the measurement of serum titers of virus-specific antibodies to SARS-CoV-2. Compared with the sampling methods required for RT-PCR, this serological assay reduces the risk of aerosol exposure, making it safer for medical staff. Therefore, anti-SARS-CoV-2 IgM and IgG antibodies have been expected to be useful as complementary tests, in addition to RT-PCR, for the diagnosis of COVID-19. However, a limitation of antibody tests is that they require a longer window period after infection than RT-PCR. Actually, the median $\operatorname{lgM}$ and IgG seroconversion period has been reported to be 10 days or longer after symptom onset ${ }^{4-6}$. However, information on the early time courses of serum antibody titers and the sensitivity and specificity of antibody tests after the onset of COVID-19 symptoms could make these tests more useful for the COVID-19.

In this study, we aimed to investigate the time courses of the anti-SARS-CoV-2 IgM and IgG titers as measured using chemiluminescent immunoassays (CLIA) and to determine the sensitivity and specificity of these tests according to the specific day after the onset of COVID-19 symptoms in Japanese subjects. 


\section{Results}

\section{Titers of SARS-CoV-2 IgM and IgG}

The time courses for the serum titers of SARS-CoV-2 IgM and IgG at each time point (number of days after symptom onset) are shown in Figure 1. Only data for which information regarding the timing of the sampling (number of days after symptom onset) were available were analyzed. As a result, the titers of SARS-CoV-2 IgM and SARS-CoV-2 IgG were analyzed in the 125 sera collected from RT-PCR-positive patients $(n=26)$ and in the 68 sera collected from RTPCR-negative patients $(n=34)$.

\section{ROC analysis and Redefinition of the cutoff values}

We performed the ROC analyses at each time point after symptom onset to determine the cutoff values (Fig. 2 and Table 2). When the ROC analyses were performed using all samples we collected ( $n=330$; RT-PCR-positive $n=186, R T-$ PCR-negative $n=144$ ), the AUC of IgM and IgG were 0.948 and 0.952 , respectively. The redefined cutoff values of both of IgM and IgG were far below the manufacturer's cutoff value, that's $0.86 \mathrm{AU} / \mathrm{mL}$ and $4.97 \mathrm{AU} / \mathrm{mL}$, respectively. When we calculated the sensitivity and specificity at these cutoff values, the sensitivity and specificity of IgM were $93.0 \%$ and $86.8 \%$, respectively. Those of IgG were $88.2 \%$ and $100 \%$, respectively.

Next, we analyzed the data only at specific points after symptom onset. the cutoff values of IgM test varied between 0.86 and $1.48 \mathrm{AU} / \mathrm{mL}$. Regarding IgG test, the AUC reached 1.00 (sensitivity:100\%, specificity:100\%) after Day 15 , and the cutoff values were equal to the border values between RT-PCR-positive and RT-PCR-negative results (Fig. 2, Table 2). These results suggested the possibility that the cutoff values could be lower than $10 \mathrm{AU} / \mathrm{mL}$ in the Japanese population: 1 or $2 \mathrm{AU} / \mathrm{mL}$ for $\mathrm{IgM}$ and $5 \mathrm{AU} / \mathrm{mL}$ for $\mathrm{lgG}$.

To validate these results, we also performed ROC analyses using randomly selected outpatients from April to June 2020 as a control group $(n=105)$ (Supplemental Fig. 1$)$. The AUCs of the IgM and IgG tests were $0.960(95 \% \mathrm{Cl}, 0.931$ $0.977)$ and $0.962(95 \% \mathrm{Cl}, 0.935-0.978)$, respectively, and the re-calculated cutoff values for both IgM and IgG were 0.89 $\mathrm{AU} / \mathrm{mL}$ (sensitivity, $92.5 \%$; specificity, $92.5 \%$ ) and $3.07 \mathrm{AU} / \mathrm{mL}$ (sensitivity, $90.9 \%$; specificity, $97.1 \%$ ), respectively.

\section{Changes in diagnostic performance after symptom onset}

We calculated the sensitivity and specificity at each cutoff value (1-10 AU/mL). Figure $3(A, B)$ shows the changes in sensitivity and specificity when the cutoff values were changed for samples obtained 9-10 days after symptom onset. Especially for IgM, when compared with a cutoff of $5-10 \mathrm{AU} / \mathrm{mL}$, the sensitivity increased up to about $80 \%$ at a cutoff of $1 \mathrm{AU} / \mathrm{mL}$. Next, we set the cutoff value for $\operatorname{lgM}$ as $1 \mathrm{AU} / \mathrm{mL}, 2 \mathrm{AU} / \mathrm{mL}$, or $10 \mathrm{AU} / \mathrm{mL}$ and that for $\operatorname{lgg}$ as $5 \mathrm{AU} / \mathrm{mL}$ or $10 \mathrm{AU} / \mathrm{mL}$ and compared the sensitivities and specificities among the time points after symptom onset. For an IgM cutoff value of $1 \mathrm{AU} / \mathrm{mL}$ (Fig. 3F), the sensitivity at 9-10 days after symptom onset (81.8\%) was much higher than that at 7-8 days after symptom onset (33.3\%). When the $\mathrm{lgM}$ cutoff value was set as $2 \mathrm{AU} / \mathrm{mL}$, the sensitivity at 9-10 days after symptom onset decreased to $54.5 \%$ (Fig. 3E). The Youden Index at cutoff values of $1 \mathrm{AU} / \mathrm{mL}$ and $2 \mathrm{AU} / \mathrm{mL}$ were 0.71 and 0.55 , respectively. For an $\mathrm{IgG}$ cutoff value of $5 \mathrm{AU} / \mathrm{mL}$, the sensitivity at $9-10$ days after symptom onset was $81.8 \%$ (Fig. 3D). When the manufacturer's cutoff value for $\mathrm{IgG}, 10 \mathrm{AU} / \mathrm{mL}$, was used, the sensitivity at the same time point decreased to $63.6 \%$ (Fig. $3 \mathrm{C}$ ). These results suggest that the serum titers of IgM and IgG after 9 days after symptom onset might have a sufficient sensitivity and specificity if the cutoff values are redefined using the Youden index for the Japanese population.

\section{Discussion}


In this study, we investigated the time courses of the serum anti-SARS-CoV-2 IgM and IgG titers and evaluated the diagnostic test performance at specific days after the onset of COVID-19. First, we confirmed the time courses of the serum antibodies. The median IgM titer increased till Day 18 after symptom onset and then declined (Fig. 1A), while that of IgG remained stable at above $400 \mathrm{AU} / \mathrm{mL}$ after Day 13 (Fig.1B). Similar dynamic changes were also reported by another study ${ }^{7}$. The timing when the median titers of IgM and IgG exceeded the manufacturer's cutoff value (10 $\mathrm{AU} / \mathrm{mL}$ ) were Day13-14 and Day9-10, respectively, which might beare similar to those for severe acute respiratory syndrome coronavirus 1 (SARS-CoV-1) ${ }^{8}$ and slightly earlier than those for Middle East respiratory syndrome coronavirus (MERS-CoV) ${ }^{9,10}$. In 14 of the 26 RT-PCR-positive patients, the seroconversion of IgG occurred earlier than that of IgM, while the seroconversion of IgM occurred earlier in one case and synchronous seroconversions were observed in 3 cases. In 8 cases, we could not determine the seroconversion timing, since both the $\operatorname{lgM}$ and IgG titers had already increased above $10 \mathrm{AU} / \mathrm{mL}$ even in the earliest available sample. In the usual immunological response to pathogens, IgM is expected to be produced earlier than IgG. Concordant with the present report, Long et al. reported ten subjects in whom the seroconversion of $\operatorname{lgG}$ occurred earlier than that of $\operatorname{IgM}^{11}$, as has also been reported in other studies conducted in China ${ }^{12-14}$. Although the exact underlying mechanism remains unclear, the earlier seroconversion of IgG might be due to the cross-reactivity of other coronavirus-specific antibodies to the target antigen used in the reagents. Actually, Pinto et al. identified a monoclonal antibody that can neutralize SARS-CoV-2 by engaging the receptor-binding domain of the spike glycoprotein from memory B cells of an individual who had suffered from SARSCoV-1 infection in $2003^{15}$. Further studies are necessary to elucidate the mechanisms responsible for the earlier seroconversion of IgG.

Next, we investigated the cutoff values and the time courses of the diagnostic abilities of serum anti-SARS-CoV-2 IgM and IgG titers. When we performed the ROC analyses using the samples at specific days after symptom onset, the AUC of IgM decreased gradually according to the time course, while that of IgG approached 1.0. These changes according to the days after symptom onset were caused by differences in the time courses of the titers; IgG continuously increased, while IgM began to decrease at around 18 days after disease onset. The IgM cutoff values calculated from the present study varied between 0.86 and $1.48 \mathrm{AU} / \mathrm{mL}$ over the days since onset, while the cutoff values for IgG were below $5 \mathrm{AU} / \mathrm{mL}$. Consequently, the cutoff values in the Japanese population could be lower ( 1 or $2 \mathrm{AU} / \mathrm{mL}$ for $\operatorname{lgM}$ and $5 \mathrm{AU} / \mathrm{mL}$ for $\mathrm{lgG})$ than the manufacturer's cutoff value $(10 \mathrm{AU} / \mathrm{mL})$. In previous studies in which the same CLIA-based assays were used, the false-positive rates for the $\operatorname{lgM}$ and IgG tests were reported to be $0 \%(0 / 33)$ and $9.1 \%(3 / 33)$ in an Italian study ${ }^{7}$ and $9.1 \%(4 / 44)$ and $0 \%(0 / 44)$ in a Chinese study ${ }^{16}$, respectively, which are somewhat higher than those in the present study $(2.9 \%[1 / 34]$ and $0 \%$ [0/34]). One of the mechanisms for false-positive results might be the presence of autoimmune diseases, and the prevalences of autoimmune diseases differ among population. For example, the prevalence of SLE, which reportedly causes false-positive cases, in Italy, China and Japan are reportedly $40-81,10-30{ }^{17}$ and $3.7-19.1^{18}$ per 100,000 of the population, respectively.

Regarding the time course of sensitivity, the diagnostic ability of antibodies, especially lgG, increases as time passes from the onset of symptoms. Zhao et al. reported that RT-PCR had a higher sensitivity (66.7\%) than IgM (28.7\%) and $\operatorname{lgG}(19.1 \%)$ within 8 days from the onset of symptoms, while the sensitivity of RT-PCR decreased to $54.0 \%$ after Day 8 and that of the antibody tests increased ${ }^{4}$. Concordantly, the diagnostic performances of both $\operatorname{lgM}$ and $\lg$ were insufficient at the time points before Day7-8 (Fig.3 C-F), while at Day9-10 we obtained much higher sensitivity when set on lower cutoff values; For IgM, the sensitivity at the cutoff value of $10 \mathrm{AU} / \mathrm{mL}, 2 \mathrm{AU} / \mathrm{mL}$ and $1 \mathrm{AU} / \mathrm{mL}$ were $36.4 \%$, $54.5 \%$ and $81.8 \%$, respectively and for $\mathrm{lgG}$, the sensitivity at the cutoff value of $10 \mathrm{AU} / \mathrm{mL}$ and $5 \mathrm{AU} / \mathrm{mL}$ were $63.6 \%$ and $81.8 \%$, respectively. These results suggested that setting the optimal cutoff value for Japanese population could make the serum antibody test more useful tool for diagnosis of COVID-19 infection, although further studies are necessary to prove this hypothesis. 
It was of note the IgG titers of all the sera collected from RT-PCR-positive patients $(n=26)$ were above 10 AU/mL at 15 days or later after symptom onset (Fig. 1B), and the sensitivity was 100\%. Several subjects with non-COVID-19 diseases, such as pneumonia caused by other pathogens or collagen diseases, can show similar clinical course and CT results, which sometimes makes a differential diagnosis difficult. The high specificity of IgG after Day 15 could help physicians to rule out COVID-19.

A limitation of this study is that only symptomatic patients were examined. Recently, the asymptomatic COVID-19 subjects have been reported to have antibody titers below the cutoff values ${ }^{19}$. According to a recent report, T cell immunity is sufficient to exclude SARS-CoV-2 in asymptomatic patients ${ }^{20}$. Therefore, in respect to infection control, which requires the screening of asymptomatic subjects, an antibody test alone might be insufficient, and combination with a PCR-based test is desirable. Nevertheless, antibody tests could help physicians to diagnose COVID-19 or nonCOVID-19 presenting with similar clinical courses among symptomatic subjects.

In summary, we investigated the time courses of the diagnostic performances of SARS-CoV-2 antibody tests in Japan and observed a high sensitivity after Days 9-10. Considering the high specificity of IgG levels at 15 days after symptom onset, the IgG test could be a very useful diagnostic tool for ruling out the possibility of COVID-19.

\section{Materials And Methods}

\section{Samples}

We collected residual serum samples remaining after routine clinical testing from 100 subjects who underwent RT-PCR testing at The University of Tokyo Hospital. Of these 100 subjects, 26 were diagnosed as having COVID-19 based on the results of RT-PCR, and the remaining 74 subjects were considered to have negative results. The characteristics of the subjects are described in Table 1. Serum specimens were stored at $-80^{\circ} \mathrm{C}$ and were centrifuged at $2,300 \times g$ for 5 minutes before measurement. The current study was performed in accordance with the ethical guidelines of the Declaration of Helsinki. Participants were informed about the study and informed consent was obtained in the form of an opt-out on the website. Patients who rejected to be enrolled in our study were excluded. The study design was approved by The University of Tokyo Medical Research Center Ethics Committee, which waived written informed consent because archived specimens were used and data in this retrospective study were retrieved from medical records (2019300NI-3).

\section{Methods}

The serum titers of SARS-CoV-2 IgM and IgG were measured using the iFlash3000 fully automatic CLIA analyzer from YHLO Biotechnology Company, Ltd. (Shenzhen, China). We used SARS-CoV-2 IgM and SARS-CoV-2 IgG kits containing magnetic beads coated with SARS-CoV-2 nucleocapsid protein and spike protein. The performance of this assay has been validated at 10 hospitals in China ${ }^{21}$. According to the manufacturer's insert, the cutoff value for the detection of both $\operatorname{lgM}$ and $\operatorname{lgG}$ is $10 \mathrm{AU} / \mathrm{mL}$.

\section{Analysis}

Receiver operating characteristics (ROC) analyses were performed using JMP PRO v15.0 (SAS Institute Inc., Cary, NC). The area under the curve (AUC) was expressed as the median and $95 \%$ confidence interval (CI). For each ROC curve, we redefined the cutoff values so that the Youden Index (= Sensitivity + Specificity -1$)$ was at a maximum. 


\section{Declarations}

The study design was approved by The University of Tokyo Medical Research Center Ethics Committee, which waived written informed consent because archived specimens were used and data in this retrospective study were retrieved from medical records (2019300NI-3).

\section{Data Availability Statement.}

The datasets generated or analyzed in the current study are available upon reasonable request.

\section{Acknowledgements}

We appreciate the Murakami Foundation's donation of the iFlash 3000 device to The University of Tokyo Hospital.

\section{Funding Sources}

None.

\section{Authorship Contributions}

Y.N. participated in the experiments and data analysis and drafted the initial manuscript; R.Y., Y.M., T.S., Y.N., and N.Y. participated in the experiments; M.K. participated in the study design, data analysis, helped to draft the manuscript, and conceived the study; C.Q., F. X., F. H., Y. K. and J. O. developed the antibody measurement system; H.O., K.M., and Y.S. participated in the discussion and helped to draft the manuscript; and T.K. and Y.Y. conceived the study, coordinated the study design, and helped to draft the manuscript. All the authors have read and approved the final manuscript.

\section{Disclosure of Conflicts of Interest}

The present study is a collaborative research project among The University of Tokyo, Shenzhen YHLO Biotech Co., Ltd, and Medical \& Biological Laboratories Co., Ltd. F. X. and F. H. are employees of Shenzhen YHLO Biotech Co., Ltd and Y. K. and J. O. are employees of Medical \& Biological Laboratories Co., Ltd.

\section{Abbreviations}

AUC, area under the curve

CLIA, chemiluminescent immunoassay

COVID-19, coronavirus infection disease 2019

MERS-CoV, Middle East respiratory syndrome coronavirus

ROC, receiver operating characteristics

RT-PCR, reverse transcriptase-polymerase chain reaction

SARS-CoV-1, severe acute respiratory syndrome coronavirus 1

SARS-CoV-2, severe acute respiratory syndrome coronavirus 2

\section{References}


1 Pascarella, G. et al. COVID-19 diagnosis and management: a comprehensive review. J. Intern. Med., doi:10.1111/joim.13091 (2020).

2 Wang, W. et al. Detection of SARS-CoV-2 in Different Types of Clinical Specimens. JAMA, doi:10.1001/jama.2020.3786 (2020).

3 Pasomsub, E. et al. Saliva sample as a non-invasive specimen for the diagnosis of coronavirus disease 2019: a cross-sectional study. Clin. Microbiol. Infect., doi:10.1016/j.cmi.2020.05.001 (2020).

4 Zhao, J. et al. Antibody responses to SARS-CoV-2 in patients of novel coronavirus disease 2019. Clin. Infect. Dis., doi:10.1093/cid/ciaa344 (2020).

5 Qu, J. et al. Profile of IgG and IgM antibodies against severe acute respiratory syndrome coronavirus 2 (SARSCoV-2). Clin. Infect. Dis., doi:10.1093/cid/ciaa489 (2020).

6 To, K. K.-W. et al. Temporal profiles of viral load in posterior oropharyngeal saliva samples and serum antibody responses during infection by SARS-CoV-2: an observational cohort study. The Lancet Infectious Diseases 20, 565-574, doi:10.1016/s1473-3099(20)30196-1 (2020).

7 Jin, Y. et al. Diagnostic value and dynamic variance of serum antibody in coronavirus disease 2019. Int. J. Infect. Dis. 94, 49-52, doi:10.1016/j.jijid.2020.03.065 (2020).

8 Hsueh, P. R., Huang, L. M., Chen, P. J., Kao, C. L. \& Yang, P. C. Chronological evolution of IgM, IgA, IgG and neutralisation antibodies after infection with SARS-associated coronavirus. Clin. Microbiol. Infect. 10, 1062-1066, doi:10.1111/j.1469-0691.2004.01009.x (2004).

9 Park, W. B. et al. Kinetics of Serologic Responses to MERS Coronavirus Infection in Humans, South Korea. Emerg. Infect. Dis. 21, 2186-2189, doi:10.3201/eid2112.151421 (2015).

10 Al Johani, S. \& Hajeer, A. H. MERS-CoV diagnosis: An update. J Infect Public Health 9, 216-219, doi:10.1016/j.jiph.2016.04.005 (2016).

11 Long, Q. X. et al. Antibody responses to SARS-CoV-2 in patients with COVID-19. Nat. Med. 26, 845-848, doi:10.1038/s41591-020-0897-1 (2020).

12 Zhang, B. et al. Immune phenotyping based on neutrophil-to-lymphocyte ratio and IgG predicts disease severity and outcome for patients with COVID-19 doi:10.1101/2020.03.12.20035048 (2020).

13 Zhang, G., Nie, S., Zhang, Z. \& Zhang, Z. Longitudinal Change of Severe Acute Respiratory Syndrome Coronavirus 2 Antibodies in Patients with Coronavirus Disease 2019. J. Infect. Dis. 222, 183-188, doi:10.1093/infdis/jiaa229 (2020).

14 Zhang, J. et al. Serological detection of 2019-nCoV respond to the epidemic: A useful complement to nucleic acid testing. doi:10.1101/2020.03.04.20030916 (2020).

15 Pinto, D. et al. Cross-neutralization of SARS-CoV-2 by a human monoclonal SARS-CoV antibody. Nature, doi:10.1038/s41586-020-2349-y (2020).

16 Infantino, M. et al. Diagnostic accuracy of an automated chemiluminescent immunoassay for anti-SARS-CoV-2 IgM and IgG antibodies: an Italian experience. J. Med. Virol., doi:10.1002/jmv.25932 (2020). 
17 Rees, F., Doherty, M., Grainge, M. J., Lanyon, P. \& Zhang, W. The worldwide incidence and prevalence of systemic lupus erythematosus: a systematic review of epidemiological studies. Rheumatology (Oxford) 56, 1945-1961, doi:10.1093/rheumatology/kex260 (2017).

18 Danchenko, N., Satia, J. A. \& Anthony, M. S. Epidemiology of systemic lupus erythematosus: a comparison of worldwide disease burden. Lupus 15, 308-318, doi:10.1191/0961203306lu2305xx (2006).

19 Zhou, F. Tracing Asymptomatic SARS-CoV-2 Carriers Among 3674 Hospital Staff. doi:https://ssrn.com/abstract=3582765 (2020).

20 Sekine, T. et al. Robust T cell immunity in convalescent individuals with asymptomatic or mild COVID-19. doi:10.1101/2020.06.29.174888 (2020).

21 Qian, C. et al. Development and Multicenter Performance Evaluation of The First Fully

Automated SARS-CoV-2 IgM and IgG Immunoassays doi:10.1101/2020.04.16.20067231 (2020).

\section{Tables}

Table 1. Characteristics of the subjects

\begin{tabular}{c|ccc}
\hline Group & Total & \multicolumn{2}{c}{ SARS-CoV-2 RNA } \\
\cline { 3 - 4 } & & Positive & Negative \\
\hline Number of subjects & & & \\
Total & 100 & 26 & 74 \\
$\mathrm{M}$ & 71 & 21 & 50 \\
$\mathrm{~F}$ & 29 & 5 & 24 \\
\hline Age [years] & 66.5 & 68.0 & 65.5 \\
(Range) & $(2-95)$ & $(24-89)$ & $(2-95)$ \\
\hline
\end{tabular}

Table 2. The time course of the diagnostic values determined with ROC analyses. 


\begin{tabular}{|c|c|c|c|c|c|c|c|c|c|c|c|}
\hline $\begin{array}{l}\text { after } \\
\text { n onset }\end{array}$ & Total & Day3- & Day5- & Day7- & Day9- & Day1 1- & Day13- & Day15- & Day17- & Day19- & Day21- \\
\hline $\begin{array}{l}\text { T-PCR } \\
\text { ositive }\end{array}$ & 186 & 186 & 183 & 174 & 165 & 147 & 120 & 86 & 67 & 54 & 43 \\
\hline $\begin{array}{l}\text { T-PCR } \\
\text { gative }\end{array}$ & 144 & 72 & 66 & 54 & 37 & 28 & 18 & 15 & 13 & 9 & 8 \\
\hline Total & 330 & 258 & 249 & 228 & 202 & 175 & 138 & 101 & 80 & 63 & 51 \\
\hline $\begin{array}{l}\mathrm{AUC} \\
5 \% \mathrm{CI}]\end{array}$ & $\begin{array}{c}0.948 \\
{[0.918-} \\
0.969]\end{array}$ & $\begin{array}{c}0.944 \\
{[0.893-} \\
0.972]\end{array}$ & $\begin{array}{c}0.944 \\
{[0.888-} \\
0.973]\end{array}$ & $\begin{array}{c}0.942 \\
{[0.874-} \\
0.975]\end{array}$ & $\begin{array}{c}0.925 \\
{[0.829-} \\
0.969]\end{array}$ & $\begin{array}{c}0.910 \\
{[0.786-} \\
0.966]\end{array}$ & $\begin{array}{c}0.931 \\
{[0.800-} \\
0.979]\end{array}$ & $\begin{array}{c}0.927 \\
{[0.762-} \\
0.980]\end{array}$ & $\begin{array}{c}0.917 \\
{[0.717-} \\
0.980]\end{array}$ & $\begin{array}{c}0.875 \\
{[0.605-} \\
0.969]\end{array}$ & $\begin{array}{c}0.849 \\
{[0.544-} \\
0.964]\end{array}$ \\
\hline $\begin{array}{l}\text { utoff } \\
\text { Value }\end{array}$ & & & & & & & & & & & \\
\hline $\mathrm{U} / \mathrm{mL}]$ & 0.86 & 0.97 & 0.97 & 0.86 & 1.00 & 1.00 & 1.48 & 1.48 & 1.19 & 1.19 & 1.19 \\
\hline $\begin{array}{l}\text { nsitivity } \\
\text { [\%] }\end{array}$ & 93.0 & 90.9 & 91.8 & 95.4 & 94.6 & 95.2 & 91.7 & 94.2 & 100 & 100 & 100 \\
\hline $\begin{array}{c}\text { ecificity } \\
\text { [\%] }\end{array}$ & 86.8 & 91.7 & 90.9 & 87.0 & 86.5 & 85.7 & 88.9 & 86.7 & 84.6 & 77.8 & 75.0 \\
\hline $\begin{array}{l}\text { AUC } \\
5 \% \mathrm{CI}]\end{array}$ & $\begin{array}{c}0.952 \\
{[0.933-} \\
0.976]\end{array}$ & $\begin{array}{c}0.958 \\
{[0.929-} \\
0.975]\end{array}$ & $\begin{array}{c}0.963 \\
0.936- \\
0.980]\end{array}$ & $\begin{array}{c}0.975 \\
{[0.950-} \\
0.988]\end{array}$ & $\begin{array}{c}0.987 \\
0.965- \\
0.995]\end{array}$ & $\begin{array}{c}0.994 \\
0.974- \\
0.999]\end{array}$ & $\begin{array}{c}0.998 \\
0.984- \\
0.999]\end{array}$ & 1.000 & 1.000 & 1.000 & 1.000 \\
\hline $\begin{array}{l}\text { Jutoff } \\
\text { Value }\end{array}$ & & & & & & & & & & & \\
\hline $\begin{array}{l}\text { ¿U/mL] } \\
\text { nsitivity }\end{array}$ & 4.97 & 4.97 & 4.31 & 4.31 & 4.31 & 4.31 & 4.31 & 3.94 & 2.95 & 2.95 & 2.95 \\
\hline $\begin{array}{l}\text { [\%] } \\
\text { ecificity }\end{array}$ & 88.2 & 88.2 & 90.2 & 93.1 & 95.8 & 97.3 & 99.2 & 100 & 100 & 100 & 100 \\
\hline [\%] & 100 & 100 & 100 & 100 & 100 & 100 & 100 & 100 & 100 & 100 & 100 \\
\hline
\end{tabular}

Figures 
Fig. 1
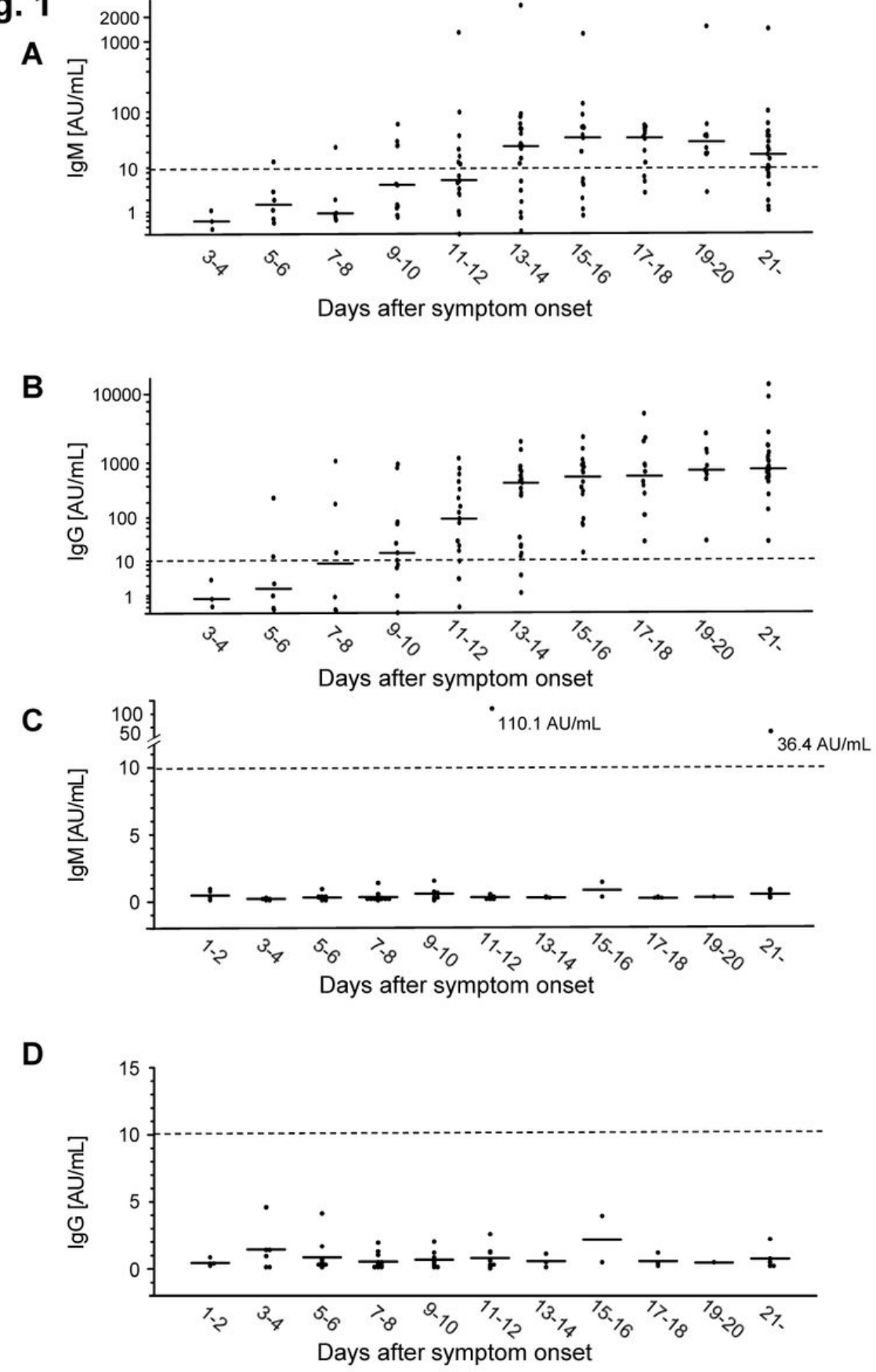

Figure 1

The time courses of serum titers of SARS-CoV-2 antibodies in COVID-19 patients after symptom onset. We measured the titers of SARS-CoV-2 IgM and SARS-CoV-2 IgG in 125 sera collected from RT-PCR-positive patients $(n=26)$ and 68 sera collected from RT-PCR-negative patients $(n=34)$. The data are plotted for days 1-2, 3-4, 5-6, 7-8, 9-10, 11-12, 13-14, $15-16,17-18,19-20$, and $\geq 21$ since symptom onset. The bars show the median titers at each timepoint. The broken line shows the manufacturer's cutoff value (10 AU/mL). A: RT-PCR-positive patients, IgM. B: RT-PCR-positive patients, IgG. C: RT-PCR-negative patients, IgM. D: RT-PCR-negative patients, IgG. 
Fig. 2

A

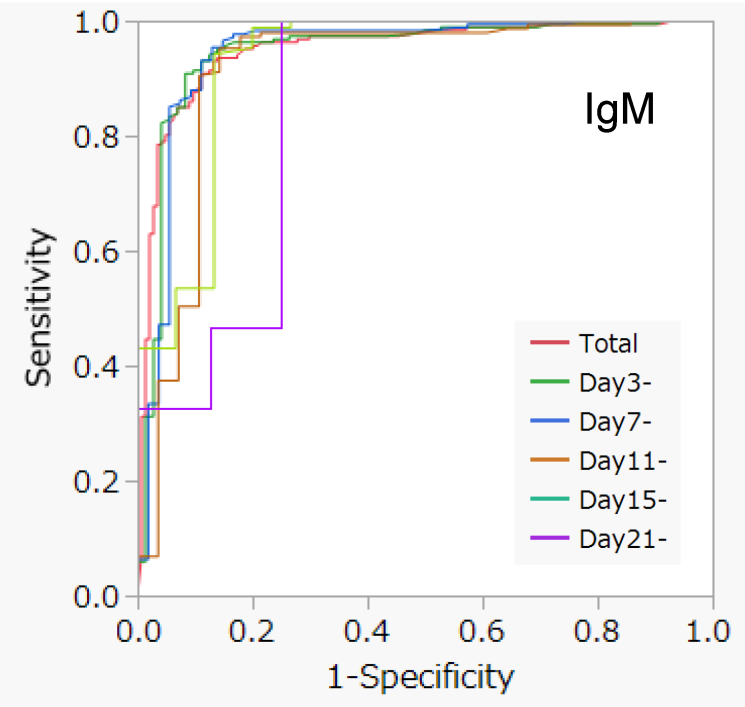

B

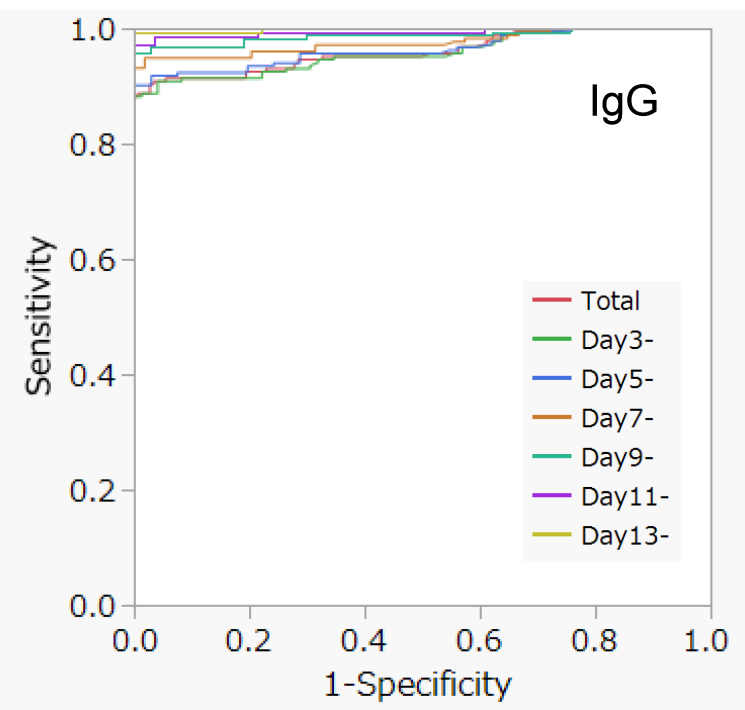

Figure 2

ROC curves for SARS-CoV-2 antibody tests. We compared the discriminating abilities of the SARS-CoV-2 IgM and SARS-CoV-2 IgG titers at different time points after symptom onset. The cutoff value for both tests was set at 10 AU/mL. A: SARS-CoV-2 IgM, B: SARS-CoV-2 IgG. 
Fig. 3
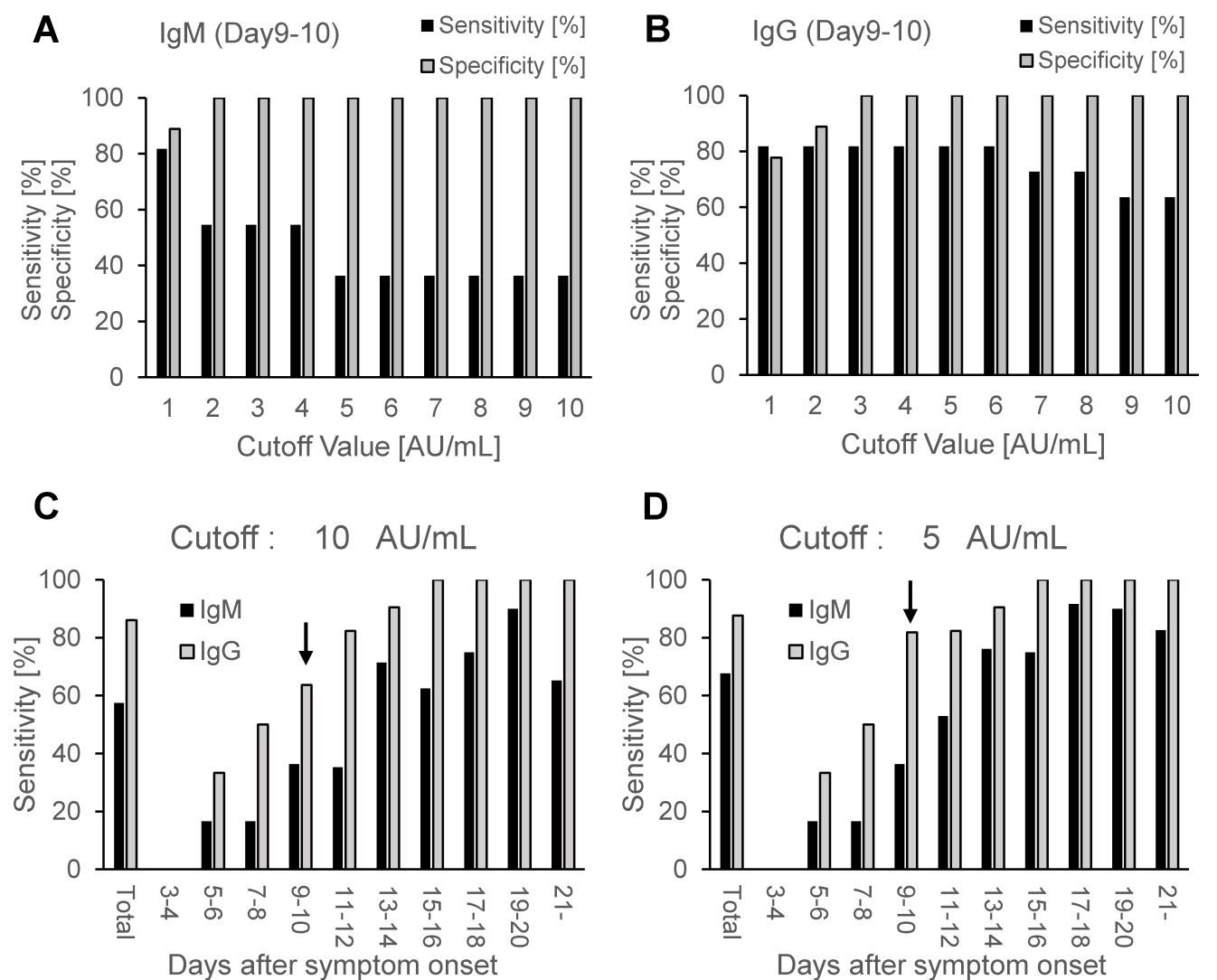

E

Cutoff : $2 \mathrm{AU} / \mathrm{mL}$

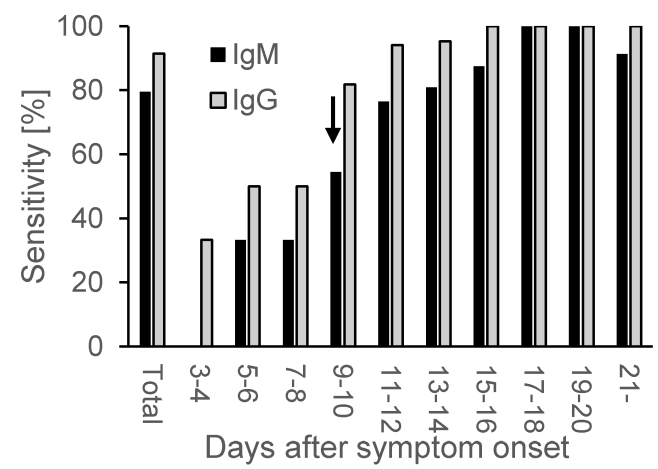

$\mathbf{F}$

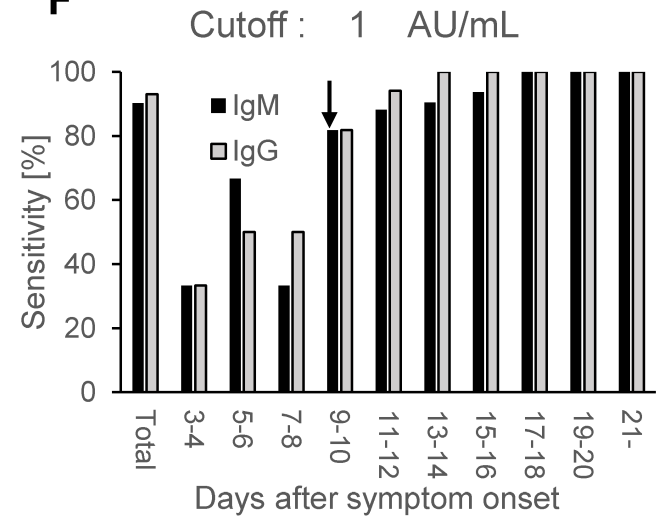

Figure 3

Diagnostic abilities of SARS-CoV-2 antibody tests according to cutoff values and time courses. We compared the sensitivities and specificities for the serum diagnosis of COVID-19 each cut off value (1 AU/mL - 10 AU/mL) at 9-10 days after symptom onset (A: SARS-CoV-2 IgM, B: SARS-CoV-2 IgG) and investigated the time course of the diagnostic ability when the cut off values were set as 1, 2, 5 and $10 \mathrm{AU} / \mathrm{mL}$ (C: $10 \mathrm{AU} / \mathrm{mL}, \mathrm{D}: 5 \mathrm{AU} / \mathrm{mL}, \mathrm{E}: 2 \mathrm{AU} / \mathrm{mL}, \mathrm{F}: 1 \mathrm{AU} / \mathrm{mL}$ ). 
Fig. 4

A

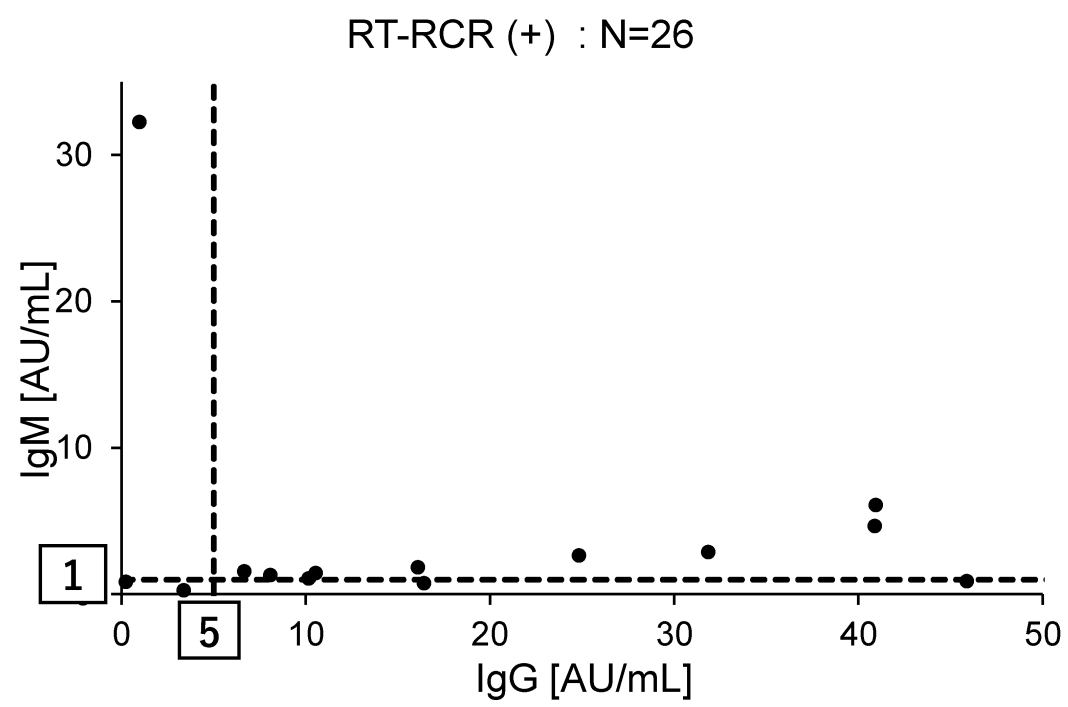

B

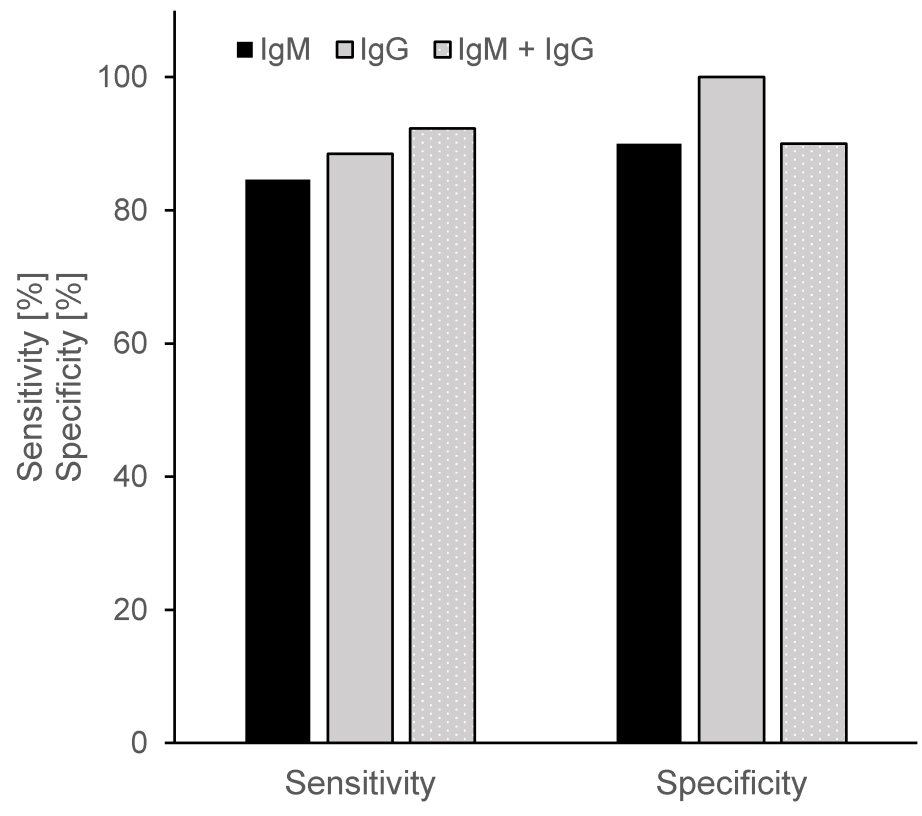

Figure 4

Diagnostic ability of the combination of $\operatorname{lgM}$ and $\lg G$. The plots of the $\lg M$ and $\lg G$ titers of sera collected from RT-PCRpositive group $(n=26)$ at 9 days or later after symptom onset are shown (A). Broken lines show the redefined cutoff values of $\lg M$ and $\operatorname{lgg}(1 \mathrm{AU} / \mathrm{mL}$ for $\operatorname{lgM}$ and $5 \mathrm{AU} / \mathrm{mL}$ for $\lg \mathrm{G})$. B: The sensitivity and specificity of $\lg M$ alone, $\lg \mathrm{G}$ alone, or the combination of IgM and IgG.

\section{Supplementary Files}

This is a list of supplementary files associated with this preprint. Click to download.

- SupplementalFigureNakano0706.pdf 